\title{
The Construction of a Reliable Multipeer Communication Protocol for Distributed Virtual Environments.
}

\author{
Gunther Stuer, Frans Arickx, Jan Broeckhove \\ University of Antwerp \\ Department of Mathematics and Computer Sciences \\ Groenenborgerlaan 171, 2020 Antwerp, Belgium.
}

gunther.stuer@ua.ac.be

\begin{abstract}
We present the design and implementation issues of a Reliable MultiPeer Protocol (RMPP). This protocol is suitable for applications in the area of distributed virtual environments and is written in Java. Motivation, protocol classification, design goals and the error recovery algorithm are discussed. This paper concludes by presenting a possible application of the RMPP.
\end{abstract}

\section{Introduction}

One of the main bottle-necks in Distributed Virtual Environments (DVE) has always been the availability of sufficient network bandwidth to allow the participating objects to communicate with each other [1]. With the introduction of multicast this problem was partly solved. But because multicast protocols are all based on best effort approaches, message delivery is not guaranteed. In order to achieve this guarantee, reliable multicast protocols were introduced $[2,3]$.

Although there are already many such protocols, none is optimized for DVEs. The problem is that multicast protocols are inherently $1 \mathrm{xN}$ protocols, i.e. one sender and many receivers. A typical DVE on the other hand has many participants, each simultaneously sending and receiving each other's information [4]. This is why DVEs rather need an $\mathrm{MxN}$ communication protocol, i.e. one with many senders and many receivers. In the literature, these protocols are known as multipeer protocols [5].

Multipeer protocols are based on a best effort approach, alike to multicast protocols. To account for guarantees on message reception, a Reliable MultiPeer Protocol (RMPP) is needed.

This paper highlights certain aspects of the development of a RMPP optimized for DVEs. In particular, the design goals, the protocol classification and the error detection algorithm are discussed. In addition a possible application of the RMPP is presented.

P.M.A. Sloot et al. (Eds.): ICCS 2002, LNCS 2330, pp. 679-686, 2002.

(C) Springer-Verlag Berlin Heidelberg 2002 


\section{Classification}

In the classification of reliable multicast protocols [6], the one presented here is most closely related to the Transport Protocol for Reliable Multicast (TRM) [7]. The RMPP is a message based protocol which means that there is no stream between sender and receiver, but rather that a number of independent messages are transmitted. Every message consists of one or more packets, each one transmitted as a UDP datagram. The most important difference with the TRM is that the RMPP is a member of the multipeer family.

Another way to classify reliable multicast algorithms is on their reliability algorithm. Initialy two groups were identified [8], sender-initiated and receiver-initiated protocols. An extension to this classification was proposed in [9] where tree-based and ring-based protocols were introduced. The most important problem with the latter two protocols is that if one node fails, many other nodes also fail. Because one of the design goals set forth in the next section will be that all nodes need to be independant of each other, tree-based and ring-based approaches are not an option for the RMPP.

In [8] it is shown that receiver-initiated protocols have much better performance than sender-initiated protocols. With receiver-initiated protocols, the receivers are responsible for detecting missing packets. They do this by sending a negative acknowledgement (NACK) to the sender whenever a missing packet is detected. On reception, the sender will re-transmit the missing packets. However, receiver-initiated protocols have two important drawbacks [9]. The first one is the danger of a NACK implosion which can happen when multiple recipients detect that a packet is missing. They will all send a NACK for the same packet with a serious regression in network performance as a result. This problem can be solved by making all receivers wait a random amount of time before actually sending the NACK. If during this delay, they receive a NACK for the same packet send by another receiver, they drop their own request. A consequence if this solution is that all NACK-requests and NACK-responses have to be multicasted to warn all interested recipients.

The second problem is that in theory these protocols need an infinite amount of memory because one can never be sure whether all receivers correctly received a certain datagram. This problem is solved heuristically by assuming that in a virtual environmnent old messages have lost their importance and can be dropped.

With sender-initiated protocols, it is the sender who is responsible for detecting errors or missing packets. To do this, the sender maintains a list of all clients and after each transmission every client has to send an acknowledgement (ACK). If one is missing, an error has occured and unacknowledged datagrams are re-transmitted. This type of protocol does not exhibit the two problems stated above, but it is not very scalable due to the large amount of ACKs.

Taking into account all the advantages and disadvantages of the mentioned protocol families, RMPP is designed to be a receiver-initiated protocol. 


\section{Design Goals}

The primary goal of this research project was the creation of a reliable multipeer system optimized for distributed virtual environments written in Java. As a reference platform we used VEplatform [10], a 100\% distributed VR-framework developed in C++ at the University of Antwerp. Note that the demand for a 100\% distributed architecture rules out the use of ring-based or tree-based reliable multicast protocols like TRAM developed by Sun Labs [11] which is a part of Sun's JRMS-project [12]. The secondary goal was to emphasise on good design. We considered the architectural aspect to be more important than top notch performance. To achieve this, we extensively used object oriented techniques, UML and design patterns.

The fact that this protocol is tuned for DVEs has three interesting consequences. The first one is that the frequency with which VR-nodes send updates has a maximum of 30 messages a second [13]. This implies that one message is sent for every screen update. When one uses dead-reckoning algorithms it is possible to reduce the update frequency to an average of 1 message per second [14]. This maximum and average value allows for the optimization of the data-structures in the reliability algorithm.

The second one is that the size of a typical VR-message is usually less than $1 \mathrm{~KB}$ because only the position, orientation and some state information is transmitted. This makes it possible to optimize buffer sizes.

The third one is that, a message is of no importance anymore after a certain amount of time because the object that sent it probably already altered one or more of the transmitted parameters. This is the reason that old messages may be dropped.

\section{Error Recovery Algorithm}

When one wants to recover from an erroneous or missing packet, it is very important to have a way to uniquely identify this packet. In the RMPP this is done in three steps. The first one is at the level of the VR-participants. Each one is identified by a unique ID, which is basically a random 32-bit number generated during construction. Every datagram transmitted will contain this ID. In this way the receiver can determine where it came from. Every datagram also contains a message sequence number (MSN) which uniquely identifies every message sent by a given participant. So, the combination (nodeID, MSN) uniquely identifies every message in the system. The third level of identification is the packetNr. This one uniquely identifies every packet within a message. As such, the 3-tuple (nodeID, MSN, packetNr) uniquely identifies every datagram in the system.

Whenever a gap is detected between messages received from the same node, or between two packets from the same message, a $N A C K$-request is sent for every message involved. When a whole message is missing, the $N A C K$-request contains its MSN, and the requested packetNr is set to 1 , since there is always at least one packet in every message. When one or more packets from a certain message are missing, the 
$N A C K$-request contains this message's $M S N$ and a list of all missing packetNrs. The sender will re-transmit all packets it receives a $N A C K$-request for. These are known as NACK-response packets. Figure 1 illustrates this algorithm.

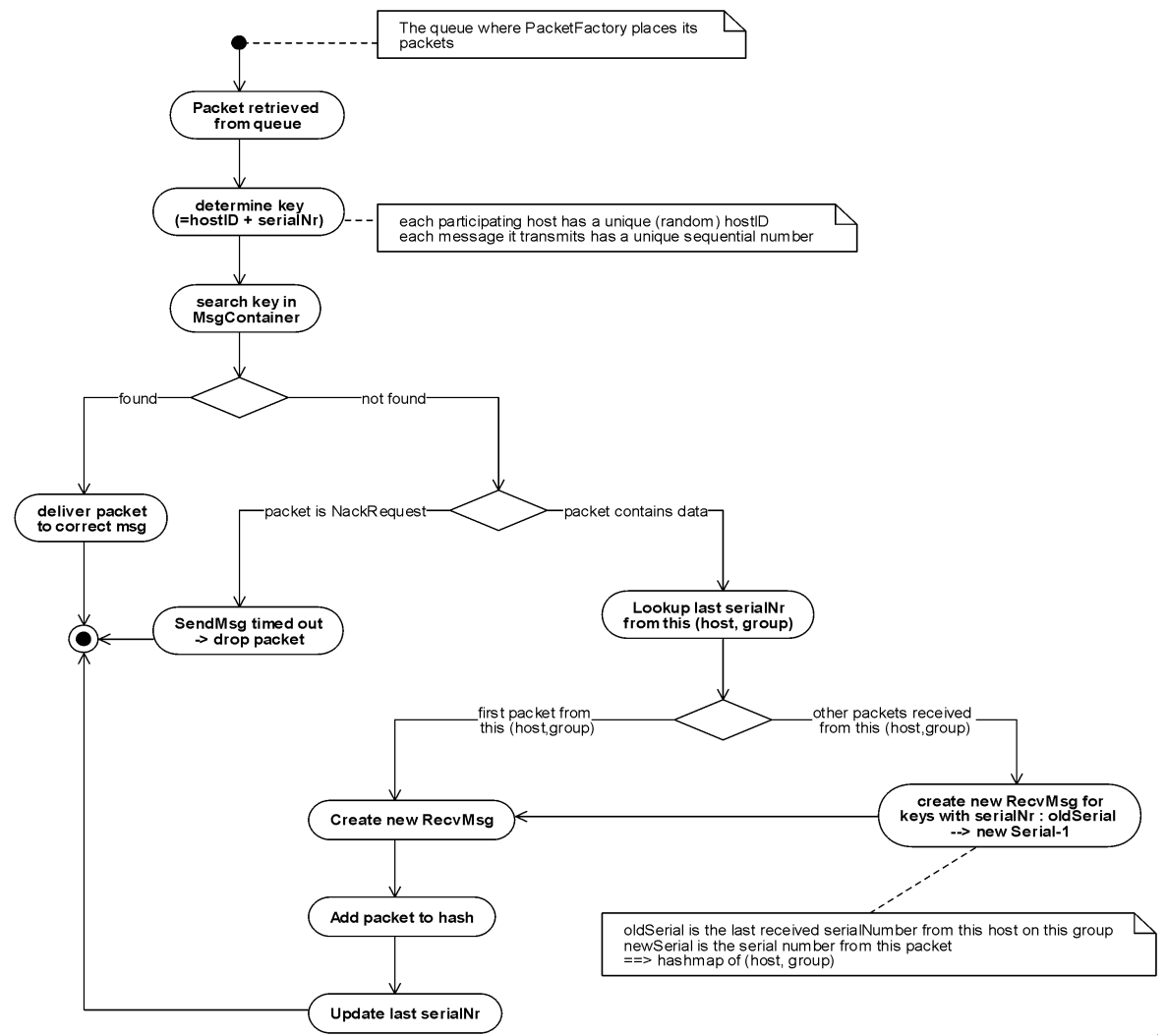

Fig. 1 This figure illustrates how messages are re-assembled at the receiver side. Every packet read from the network is stored in a queue until there are enough resources available to process it. When a packet is the first one for a particular message, the message is created. All other packets are routed towards it based on their key-value which is computed using the packet's unique ID.

When the first packet of a given message arrives, the latter is created at the receiver side and its timer is set to receiveTimeout. Whenever another packet for this message arrives, it is added to the message and the timer is reset. When it reaches zero, the RMPP assumes all unaccounted packets lost and sends a NACK-request for them. If however all packets were received, the message is considered to be complete.

When a $N A C K$-request is sent, the timer of the error producing message is set to nackTimeout. If no NACK-response is received before the timer ends, another $N A C K$-request is sent. This goes on until maxRequests NACK-requests have been transmitted after which the message is considered lost and removed from the system. If on the other hand a NACK-response is received, the timer is reset to recvTimeout and the algorithm starts all over. Figure 2 illustrates this. 


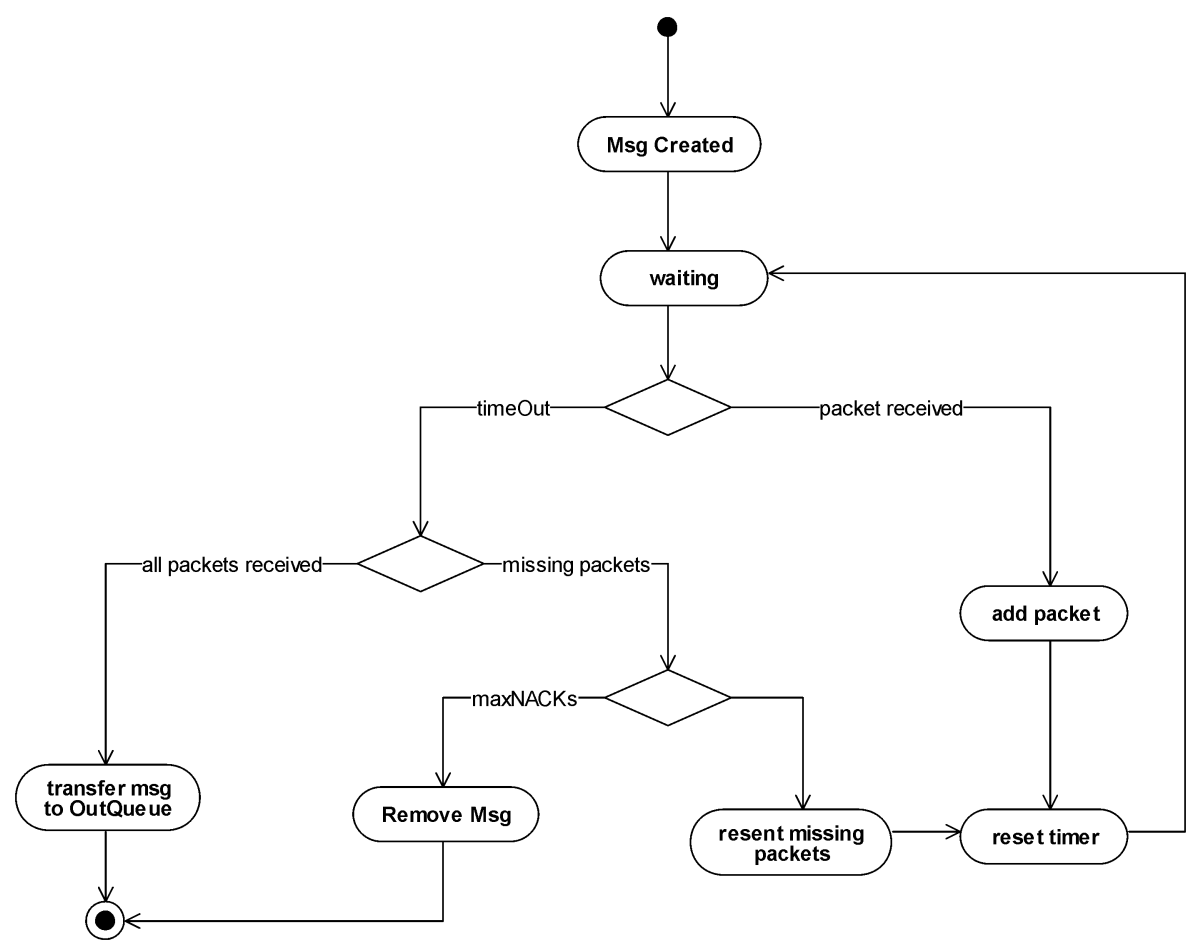

Fig. 2 This figure describes the use of timers when receiving a message. When the message is created and whenever a new packet for it arrives, the timer is reset to receiveTimeout. If it times out, the message checks whether it is complete. If not, it checks whether is can send another NACK or whether it should drop this message. If the first option is chosen, a NACK is sent for all missing packets and the timer is reset to NackTimeout.

The sender keeps every message in memory for sendTimeout seconds. This timer is reset with every incoming $N A C K$-request. When it reaches zero, the sender assumes that all receivers did receive the message correctly and removes it from memory. This is illustrated in figure 3.

Java provides no guarantees when it comes to real-time concepts. And indeed, in the first version of the RMPP a major problem was that many timeouts were missed because the Java virtual machine was performing background tasks. To resolve this problem all timeouts mentioned in the previous paragraphs have been made dynamic. Whenever one is missed, its value is increased and whenever one is met with enough spare time, its value is decreased. This way the RMPP assures vivid responses, while still adapting to the characteristics of the network, hardware and underlying operating system.

By taking into account the fact that most VR-messages are small and fit in a single datagram, an important optimization can be made. On reception of such a message, the recvTimeout notification can be omitted since we already have the complete message. 


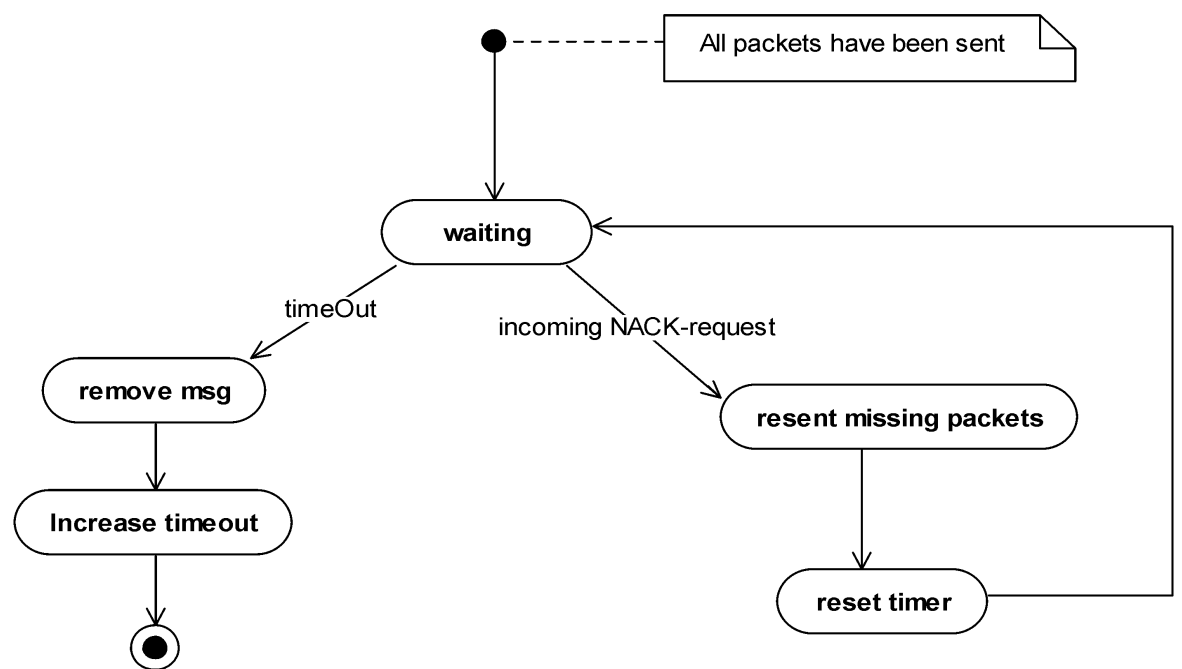

Fig. 3 This figure shows the use of timers to send a message. When all packets are transmitted, a timer is set to sendTimeout milliseconds. When it expires, the message is considered to be deliverd and removed from memory. If on the other hand a NACK-request is received, all requested packets are retransmitted and the timer is reset.

By doing this, the protocol uses much less resources to handle the same amount of messages.

\section{A Possible Application}

In this section a possible application of the RMPP is discussed. It is a component of the highly dynamical DVE our research group is considering to construct.

Object mirroring is a possible strategy to minimize communications in a distributed virtual environment. One can use the RMPP to multicast the VR-objects to all interested nodes. With the use of Java classloading it is possible to create a local object from this stream of bytes. This local object serves as a proxy for the original one. Figure 4 illustrates this principle where object A gets replicated to objects A' at the receiver sides.

All communication between the original object and its proxies also relies on the RMPP system. When an object wants to update its proxies, an XML message is transmitted which consists of two parts. The first one identifies the target object and the second part contains the actual message.

Using this technique, the virtual world is extremely expandable because each type of object (e.g. table, avatar, room, ...) can define its own message scheme. As such, one does not need to specify the complete protocol from the very beginning. 


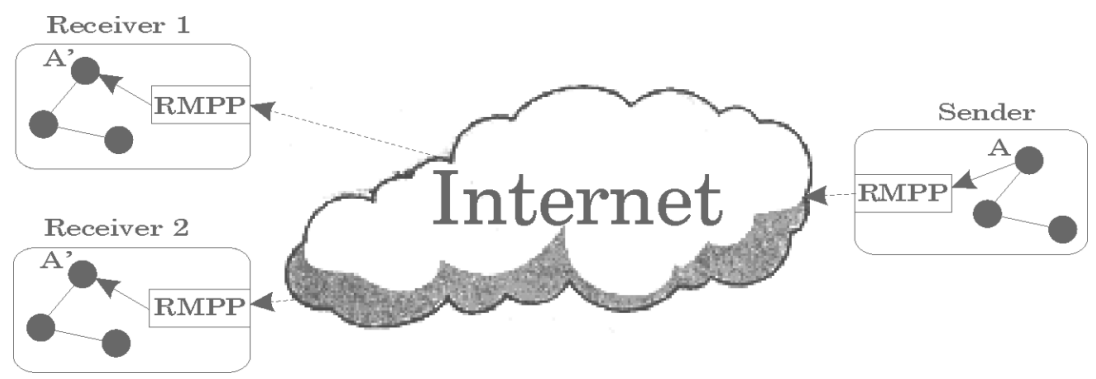

Fig. 4 VR-Object Mirroring.

\section{Conclusions}

Our contribution in this paper has been to develop a Reliable MultiPeer Protocol (RMPP) with a weak model of membership similar to the one used in IP Multicast. This protocol is geared towards applications in the area of multi-user DVEs. To achieve our design goals - in particular scaleability and a 100\% distributed architecture - a receiver initiated protocol was used.

The presented algorithm is capable of reliably delivering a series of messages conforming to the multipeer communications paradigm. One of the challenging problems in this research was the fact that Java is not tuned towards real-time applications. The current RMPP algorithm account for this problem.

\section{References}

1. Zyda, M.J.: Networking Large-Scale Virtual Environments. Proceedings of Computer Animation 1996.

2. Hall K.A.: The implementation and evaluation of reliable IP multicast. Master of Science Theses, University of Tennessee, Knoxville, USA, 1994.

3. Birman K.P.: A Review of experiences with reliable multicast. Software - Practise and Experience, 1999, Vol 29, No. 9, pp 741-774.

4. Sato F., Minamihata K., Fukuoka H., Mizuno T.: A reliable multicast framework for distributed virtual environments. Proceedings of the 1999 International Workshop on Parallel Processing.

5. Wittmann R., Zitterbart M.: Multicast Communications. Academic Press, 2000, chapter 2 . 
6. Obraczka K.: Multicast Transport Protocols: A survey and taxonomy. IEEE Communications Magazine, 1998, pp. 94-102.

7. Sabata B., Brown M.J., Denny B.A.: Transport Protocol for Reliable Multicast: TRM. Proceedings of IASTED International Conference on Networks, 1998, pp. $143-145$.

8. Pingali S.: A comparison of sender-initiated and receiver-initiated reliable multicast protocols. Performance evaluation review, Vol. 22, pp 221-230, 1994.

9. Brain N.L., Garcia-Luna-Aceves J.J.: A Comparison of known classes of reliable multicast protocols. MS thesis, University of California, 1996.

10. Demuynck K.: The VEplatform for distributed virtual reality. Ph.D. thesis, University of Antwerp, Belgium, 2000.

11. Chiu D.M., Hurst S., Kadansky M., Wesley J.: TRAM: A tree-based reliable multicast protocol. Sun Research Labs Technical Report TR-98-66.

12. Hanna S., Kadansky M., Rosenzweig Ph.: The Java Reliable Multicast Service: A Reliable Multicast Library. Sun Research Labs Technical Report TR-98-68.

13. Brutzman D.P., Macedonia M.C., Zyda M.J.: Internetwork Infrastructure Requirements for Virtual Environments. Proceedings of the 1995 symposiom on Virtual Reality Modeling Languages.

14. Demuynck K., Arickx F., Broeckhove J.: The VEplatform system: a system for distributed virtual reality. Future Generation Computer Systems. No 14, 1998, pp. 193-198. 